\title{
A SOLID STATE RARE GAS HALIDE LASER: XeF IN CRYSTALLINE ARGON
}

\author{
N. SCHWENTNER ${ }^{1}$ \\ Institut für Atom- und Festkörperphysik, Freie Universität Berlin, D-1000 Berlin 33, Germany \\ V.A. APKARIAN ${ }^{2}$ \\ Institute for Surface und Interface Science, Department of Chemistry, University of Californiu, Irvine, CA 92717, USA
}

Received 15 October 1988; in final form 1 December 1988

\begin{abstract}
Optically pumped laser action has been achieved over the $\mathrm{XeF}(\mathrm{B} \rightarrow \mathrm{X})$ and $(\mathrm{C} \rightarrow \mathrm{A})$ transitions in crystalline argon. Conversion efficiencies as high as $30 \%$ are observed. Gain measurements indicate that the laser should be tunable over the broad $\mathrm{C} \rightarrow \mathrm{A}$ band of $\approx 80 \mathrm{~nm}$.
\end{abstract}

\section{Introduction}

We report the first demonstration of an optically pumped visible laser in a rare gas crystal. The system, XeF in crystalline argon, is representative of the large family of solid state rare gas halide exciplexes which contains members suitable as laser candidates in the visible, UV and VUV spectral ranges.

The rare gas crystals used in the present work were prepared in the apparatus used by Schwentner and coworkers in their effort to develop solid state VUV excimer lasers [1]. By exploiting the high excimer densities attained in electron-beam-pumped rare gas crystals, they achieved very high radiant powers $\left(\approx 10^{6} \mathrm{~W}\right.$ ) at 128,148 and $172 \mathrm{~nm}$ and inferred gain coefficients of $10 \mathrm{~cm}^{-1}$. However, the achievement of overall positive gain failed in those systems due to transient absorptions and scattering losses from electron-beam-induced defects [1]. In contrast with electron beam pumping, recent observations of stimulated emission at $5 \mu \mathrm{m}$ in $\mathrm{CO}$-doped $\mathrm{N}_{2}$ crystals grown by the same apparatus, served to demonstrate that the optical quality, at least in the infrared spectral range, of these crystals are maintained under optical pumping conditions [2]. The results to be reported here, clearly demonstrate that the same

1 Research carried out during sabbatical stay at Irvine.

2 To whom correspondence should be addressed. crystals are also well suited as laser media in the visible spectral range.

The favorable photodynamical parameters of rare gas halide exciplexes for laser applications are well established and reflected in the fact that they form the most commonly used gas phase UV lasers [3]. In a recent series of investigations, Apkarian and coworkers undertook the study of photodynamics in condensed phase rare gas halides - in both solid and liquid rare gases [4]. Most pertinent to the present are their solid state results. They showed that rare gas solids doped with atomic halogens could be prepared by in situ photolysis of molecular halogens and hydrogen halides [4,5]. Furthermore, they noted that such solids are ideally suited as optically pumped laser media since high densities of rare gas exciplexes $\left(\mathrm{Rg}^{+} \mathrm{X}^{-}\right.$in doubly doped systems and $\mathrm{Rg}_{2}^{+} \mathrm{X}^{-}$in a single rare gas host) could be generated, and the nonradiative decay mechanisms present in the gas phase are effectively eliminated. Indeed very large gain coefficients were demonstrated over the $\mathrm{Xe}_{2}^{+} \mathrm{Cl}^{-}\left(4^{2} \boldsymbol{\Gamma} \rightarrow 1^{2} \boldsymbol{\Gamma}\right)$ transition in $\mathrm{Cl}$-doped thin films of solid xenon [6]. Halogen-doped rare gas crystals was the logical sequence for testing the viability of a solid phase exciplex laser.

Related to these studies are the liquid phase rare gas exciplexes. Rhodes and coworkers have already demonstrated laser action over the XeF $(B \rightarrow X)$ transition at $404 \mathrm{~nm}$ by optically pumping $F_{2}: X e: A r$ 
solutions [7]. Electron-beam-pumped liquid phase rare gas exciplex lasers have also been realized [8]. In the case of liquids, the usual optical pumping mechanism relies on two-photon charge transfer transitions for which high pump power densities are required. The attainable exciplex number densities in liquids is limited by the damage threshold of window materials, and nonradiative quenching of the exciplexes due mainly to exciplex-halogen donor collisions [9]. Problems associated with windows are eliminated by resorting to free standing crystals. Moreover, spatial localization in solids, precludes exciplex-exciplex and exciplex-halogen donor quenching channels. In the present system, the longterm degradation channel associated with the formation of stable covalent xenon fluorides (such as $\mathrm{XeF}_{2}$ ) is also absent.

The aims of this investigation were: to achieve laser action in a halogen-doped rare gas crystal; to demonstrate that doped free standing crystals of sufficient optical quality could be grown for this purpose by standard techniques; to test the predicted efficiencies of the photochemistry of production and population of the exciplexes; to evaluate the yield in the extraction of the stored energy; and finally to test the possibility of tunability on the broad bound-torepulsive bands of exciplexes. Initial experiments in $\mathrm{Cl}$-doped xenon crystals were not successful. In these dilute crystals, and at the high power densitics necessary for laser action, a transient absorption over a broad wavelength range $(\approx 200 \mathrm{~nm}$ broad centered at $590 \mathrm{~nm}$ ), which is attributed to the xenon host, prevented positive gain [10]. Hence the choice of the doubly doped argon host, $\mathrm{F}_{2}: \mathrm{Xe}: \mathrm{Ar}$, in which $\mathrm{XeF}$ could be prepared by photodissociation of $\mathrm{F}_{2}$. The spectroscopy of $\mathrm{XeF}$ in argon matrices has previously been studied [11]. The $B \rightarrow X$ transition centered at $411 \mathrm{~nm}$ corresponds to a bound-bound transition and shows a vibrational progression. While broadened by strong coupling to the host, the widths associated with individual transitions are narrow in comparison with typical bound-repulsive exciplexic emissions, such as the $C \rightarrow A$ band in the same system. The relaxation of both $B$ and $C$ states is radiative, with lifetimes of 6 and $120 \mathrm{ns,}$, respectively [ 11 ]. The relatively narrow linewidths in the $B \rightarrow X$ emission progression, coupled with the short lifetime of the B state, lead to a very large stimulated emission cross section making this transition well suited for a proof-of-principles demonstration. Laser action was also anticipated on the $\mathrm{C} \rightarrow \mathrm{A}$ transition despite the nearly two orders of magnitude smaller gain cross section due to its longer lifetime and broader lineshape. The latter expectation is based on the large number densities of exciplexes attainable in crystals doped with atomic halogens. The visibility of the purple $B \rightarrow X$ and green $C \rightarrow A$ bands adds to the convenience of this system for a case study.

\section{Experimental}

The device used for growing free standing Ar crystals doped with $\mathrm{Xe}$ and $F_{2}$ has been previously described in some detail $[1,2]$. A plexiglass box of $20 \times 6 \times 10 \mathrm{~mm}^{3}$ volume is pressed against a copper block held at $20 \mathrm{~K}$ by a closed cycle refrigerator. The crystal is grown in the box in $\approx 15 \mathrm{~min}$ by deposition of the gas mixture at a pressure of 100-200 Torr. The cryostat is then raised until the crystal stands free in the vacuum of the chamber.

Several crystals with different dopand concentrations were prepared. Mixtures of $\mathrm{F}_{2}: \mathrm{Xe}$ : Ar with relative partial pressures of $1: 1: 100$ yielded crystals of poor optical quality, presumably due to the high concentrations of xenon atoms. Crystals of $1: 1: 1000$ composition were essentially transparent with only a slightly hazy appearance. However, the penetration depth of $\approx 1 \mathrm{~mm}$ of the pump light, after extensive irradiation of the solid to generate $\mathrm{XeF}$ centers, was too short for the longitudinal pumping geometry along the $6 \mathrm{~mm}$ thickness of the crystal. Optically clear crystals could be prepared for compositions of $1: 1: 3000$. Photoconversion of $F_{2}$ to $\mathrm{XeF}$ leads to a cone of absorbing centers with a volume of $2 \times 0.5 \times 6 \mathrm{~mm}^{3}$ determined by the pump geometry; see fig. 1 . The excitation density along the cone was visually even, indicating a moderate attenuation of the pump beam. The cone, however, remains optically clear for visible radiation. Due to scattering of radiation at the crystal/vacuum interfaces, the propagation of the pump beam in the crystal could easily be located. Scattering at the surfaces in the case of a transmitted $\mathrm{He}$ : $\mathrm{Ne}$ laser beam appear as weak haloes. The surface quality of the crystal is determined by the finish on the walls of the box, and is some- 


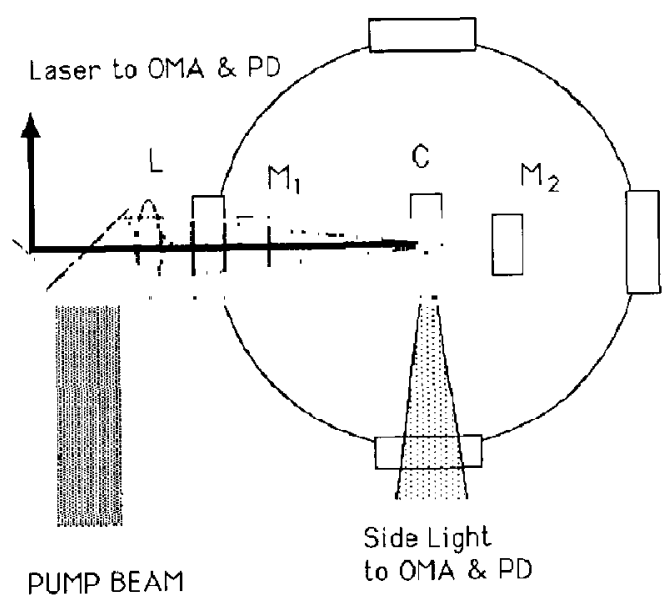

Fig. 1. Experimental diagram. The free standing crystal (C) and cavity mirrors ( $M 1$ and $M 2$ ) are contained in the vacuum chamber. The crystal is pumped with a gas phase $\mathrm{XeF}$ laser, which is focused on the crystal with a $20 \mathrm{~cm}$ focal length lens (L) from outside the chamber. Fluorescence is monitored as side-light while the on-axis beam is monitored through appropriate filters. The time evolution is monitored with a photodiode (PD) while the spectral distributions are recorded with an optical multichannel analyzer (OMA).

what degraded during the extraction process. Scattering at the crystal surfaces leads to a significant loss mechanism in the resonator, limiting the total number of round-trip passes in the cavity.

A gas phase XeF laser (Lambda Physik EMG 201) operating at $351 \mathrm{~nm}$ is used as the pump source. The pump beam is focused by a $5 \mathrm{~cm}$ diameter, $20 \mathrm{~cm}$ focal length lens along the longitudinal axis of the cavity (fig. 1). The cavity is formed by a pair of plane dielectric mirrors of $1.25 \mathrm{~cm}$ diameter installed in the vacuum chamber. The mirror on the pump side is placed at $7 \mathrm{~cm}$ from the crystal, while the output coupler is placed at a distance of $2 \mathrm{~cm}$ on the opposite side. They are mounted on a bellows assembly that allows for alignment via external micrometers. Both mirrors have transmissions of $84 \%$ at $351 \mathrm{~nm}$, and reflectivity of $30 \%$ at $410 \mathrm{~nm}$. The pump mirror is coated for maximum reflectivity ( $>99.9 \%$ ) at 500 $\mathrm{nm}$, its reflectivity at $530,540,550$ and $600 \mathrm{~nm}$ is $99.7 \%, 97 \%, 62 \%$ and $27 \%$, respectively. The output coupler mirror was coated for $95 \%$ reflectivity at 532 $\mathrm{nm}$. Its reflectivity at the same wavelengths was $94 \%$, $93.4 \%, 92 \%$ and $61 \%$.
The time evolution and the wavelength distribution of the emitted light is monitored either along or perpendicular to the laser axis. They are alternatively recorded either by a fast Si PIN photodiode combined with a $100 \mathrm{MHz}$ digitizing scope (Tektronix 2430 ), or a $0.25 \mathrm{~m}$ monochromator equipped with gratings of 1200 and 140 grooves $/ \mathrm{mm}$ and a gated intensified diode array (OMA III).

\section{Results}

The results reported here are exclusively from a sample with original composition of 1:1:3000, $\mathrm{Xe}: \mathrm{F}_{2}$ : Ar. Since the $F_{2}$ absorption cocfficient [12] at $351 \mathrm{~nm}$ is $\approx 1 \times 10^{-21} \mathrm{~cm}^{2}$, the crystal is originally nearly transparent to the pump laser. With irradiation, the XeF B and $C$ emissions develop and the pump beam is attenuated. The growth of the XeF population was followed by monitoring the fluorescence intensity as a function of time. After several thousand pulses the photoconversion is complete, i.e. a plateau is reached in the growth curve of the exciplexic emission intensity.

Fluorescence spectra obtained at low excitation powers and in the absence of cavity mirrors are shown in figs. 2a and $3 a$. The $411 \mathrm{~nm}$ band corresponds to the bound-bound $\mathrm{B}(1 / 2) \rightarrow \mathrm{X}{ }^{2} \Sigma^{+}$transition, and shows a vibrational progression which is better resolved in fig. $2 a$ than in ref. [11]. A direct measure of the B state lifetime was not possible in our studies due to the $20 \mathrm{~ns}$ width of the excitation pulse. However, our results are consistent with the 6 ns lifetime measured in ref. [11] for $\operatorname{XeF}(B)$ in Ar. The broad band centered at $536 \mathrm{~nm}$ is identified as the diatomic $\mathrm{XeF} \mathrm{C}(3 / 2) \rightarrow \mathrm{A}^{2} \Pi$ transition. Both transitions are red-shifted relative to their respective gas phase values due to solvation of these ionic states in the dielectric of the host. The $\mathrm{C} \rightarrow \mathrm{A}$ assignment has been doubted previously due to the large variation in observed emission intensities of $B$ and $C$ states in matrices prepared from different fluorine donors [11]. Indeed we do observe a variation in $B: C$ intensity ratios as a function of the extent of the photoconversion. The ratio of $B: C$ emission maxima increases from the initial value of $1: 1$ to $3: 1$ after extensive irradiation of the solid. In $\mathrm{F}_{2}$ : Xe: Ar samples of $1: 1: 100$, the ratio at early irradiation times 


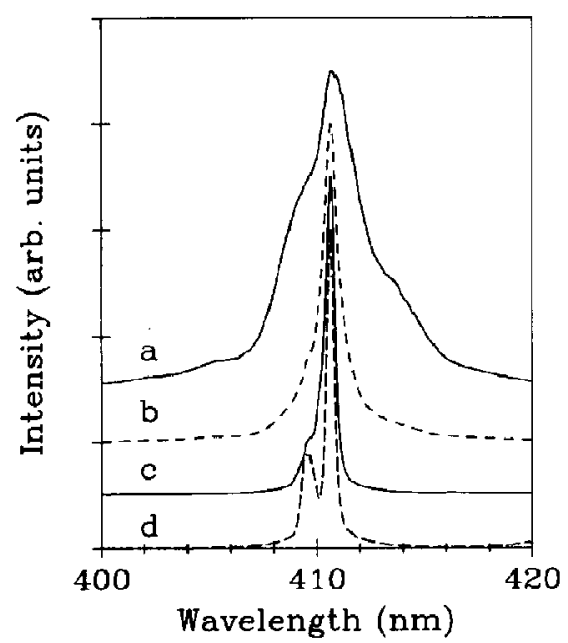

Fig. 2. Normálized emission spectra of $\operatorname{XeF}(B \rightarrow X)$ transition in solid argon. (a) Fluorescence spectrum, collected as side-light under low-energy pumping conditions, shows a progression originating from $v^{\prime}=0$ of the B state. (b) On-dxis tnission showing line narrowing due to stimulated emission at a pump energy of $18 \mathrm{~mJ}$. (c) Lasing on the largest member of the progression at 44 $\mathrm{mJ}$ of pump energy. (d) On-axis emission showing laser action on both $v^{\prime}=0$ and 1 levels of the B state at a pump energy of 44 mJ.

begins at $1: 2$. Excitation at $351 \mathrm{~nm}$ leads mainly to absorption on the XeF $(B-X)$ transition. The $C$ state is populated by internal conversion from the $B$ state. Although the exact mechanism is not well understood, we speculate that the initial branching ratio is influenced by the vicinity of $F$ or $F_{2}$ species at the photogenerated $\mathrm{XeF}$ centers. The $\mathrm{C}$ state lifetime of $120 \mathrm{~ns}$ is in agreement with that of ref. [11]. Given the lifetimes and lineshapes, stimulated emission cross sections, $\sigma_{\mathrm{s}}$, of $10^{-13}$ and $10^{-17} \mathrm{~cm}^{2}$ can be derived for the $B \rightarrow X$ and $C \rightarrow A$ transitions in solid Ar (table 1). The overall C: $B$ intensity ratios, areas of time-integrated fluorescence bands, start at 20:1 and reach a ratio of $\approx 7: 1$ at completion of the photocunversion. The steady state $B$ population is further reduced by a factor of 3 due to its short lifetime in comparison to the pump pulse duration. These steady state population ratios, $\gamma$, are given in table 1 . The product $\gamma \sigma_{\mathrm{s}}$, which determines the relative amplification coefficients, favors $B$ by a factor of 1.7 to 3.3 depending on the extent of conversion (table 1 ).

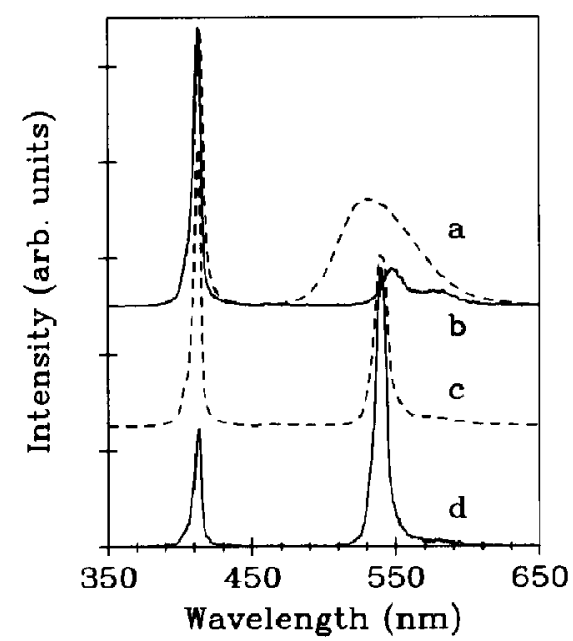

Fig. 3. Normalized emission spectra of XeF $(B \rightarrow X)$ and $(C \rightarrow A)$ transitions centered at 411 and $536 \mathrm{~nm}$, respectively. (a) Sidelight fluorescence. (b) On-axis emission at a pump energy of 12 $\mathrm{mJ}$. The degraded $\mathrm{C} \rightarrow \mathrm{A}$ band is due to the dielectric coating of the cavity mirror ( $R \geqslant 99.9 \%$ at $500 \mathrm{~nm}$ ). (c) Lasing on both bands at a pump energy of $51 \mathrm{~mJ}$, condition under which the $B \rightarrow X$ laser is more intense. (d) Laser action at $76 \mathrm{~mJ}$ of pump energy, shows saturation of the $B \rightarrow X$ laser. The $C \rightarrow A$ laser is now more intense.

The difference between $B$ and $C$ is not very large, therefore the resonator reflectivities should determine the transition to be amplified. Note, however, that a factor of 20 more power should be extractable from the $\mathrm{C}$ state due to its larger population.

A series of gain measurements were performed prior to installation of the cavity mirrors. This was achieved by synchronizing the output of a pulsed dye laser with the pump laser and overlapping the two beams in the crystal in a counterpropagating geometry. Small signal amplification of greater than $100 \%$ was observed at all studied wavelengths, from 520 to $590 \mathrm{~nm}$ over the $\mathrm{C} \rightarrow \mathrm{A}$ band, and at several wavelengths near the maximum of the $B \rightarrow X$ transition [10]. Amplification by a factor $\geqslant 10$ was obscrved at the emission maxima of both $B$ and $C$ states. With $8 \mathrm{~mJ}$ of pump power, up to $30 \%$ of the deposited photons could be extracted near the $\mathrm{C} \rightarrow \mathrm{A}$ band maximum. During these measurements amplified spontaneous emission was evidenced by line-narrowing along the pump direction. 
Table 1

Spectroscopic parameters of $\mathrm{XeF}$ in crystalline argon. The $B \rightarrow X$ parameters are for the vibronic transition at $411 \mathrm{~nm}$. $\tau$ is the radiative lifetime; $\sigma_{\mathrm{s}}$ is the stimulated emission cross section; $\gamma$ is the B:C population ratio (normalized to $C$ ), "early" and "late" refer to the extent of photoconversion; $\alpha$ is the gain coefficient; and $l$ is the length of the active medium

\begin{tabular}{|c|c|c|c|}
\hline & \multirow{2}{*}{$\begin{array}{l}\text { Pump } \\
\text { energy } \\
(\mathrm{mJ})\end{array}$} & \multicolumn{2}{|l|}{ Transition } \\
\hline & & $\mathbf{B} \rightarrow \mathbf{X}$ & $\mathrm{C} \rightarrow \mathrm{A}$ \\
\hline \multicolumn{2}{|l|}{$\tau(\mathrm{ns})$} & 6 & 120 \\
\hline \multicolumn{2}{|l|}{ fwhm (nm) } & 2.4 & 64 \\
\hline \multicolumn{2}{|l|}{$\sigma_{\mathrm{s}}\left(\mathrm{cm}^{2}\right)$} & $10^{-15}$ & $10^{-17}$ \\
\hline \multicolumn{2}{|l|}{$\gamma$ early } & $1.7 \times 10^{-2}$ & 1 \\
\hline \multicolumn{2}{|l|}{ late } & $3.3 \times 10^{-2}$ & 1 \\
\hline \multicolumn{2}{|l|}{$\gamma \sigma_{s}\left(\mathrm{~cm}^{-2}\right)$ early } & $1.7 \times 10^{-17}$ & $10^{-17}$ \\
\hline \multicolumn{2}{|l|}{ late } & $3.3 \times 10^{-17}$ & $10^{-17}$ \\
\hline \multirow[t]{4}{*}{ fwhm $(\mathrm{nm})$} & 29 & 0.6 & \\
\hline & 44 & 0.4 & \\
\hline & 51 & & 10.6 \\
\hline & 76 & & 8.8 \\
\hline \multirow[t]{4}{*}{$\alpha_{0} l$} & 29 & 16 & \\
\hline & 44 & 36 & \\
\hline & 51 & & 37 \\
\hline & 76 & & 53 \\
\hline$\alpha_{\max }\left(\mathrm{cm}^{-1}\right)$ & & $170-330$ & 100 \\
\hline
\end{tabular}

When the crystal is pumped with cavity mirrors in place, both green $(C \rightarrow A)$ and purple $(B \rightarrow X)$ laser beams are observed along the cavity axis, in both directions. The divergences of the observed beams of $\approx 200 \mathrm{mrad}$ closely follow the excited volume dimensions. When a fresh spot is irradiated, lasing starts on the green band, which is preferred by the cavity reflectivity. With extended irradiation near the threshold of the green laser, the purple beam switches on, due to the increase in the $B / C$ ratio. The intensity in the amplified beams increase exponentially with pump energy. An increase of more than four orders of magnitude is observed in the amplified purple beam, for an increase in the pump energy from 2.5 to $25 \mathrm{~mJ}$. Saturation is observed near this upper limit. The $\mathrm{C} \rightarrow \mathrm{A}$ lifetime is dramatically shortened and becomes indistinguishable from the temporal profile of the pump beam above $50 \mathrm{~mJ}$, see fig. 4 . Lifetime shortening of the $B$ state is less obvious due to its short radiative relaxation time in comparison to the pump pulse width.

Lifetime shortening is accompanied by a narrowing of the emission bands. A sequence of normalized

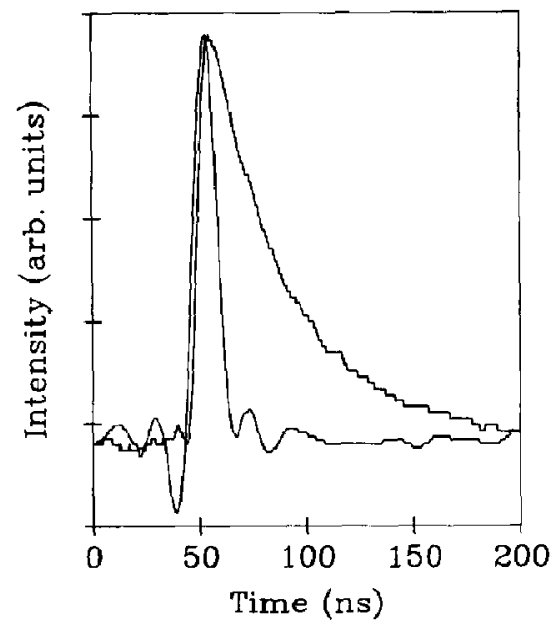

Fig. 4. Time evolution of the $\mathrm{C} \rightarrow \mathrm{A}$ emission. Shown are the sidelight fluorescence (averaged over 8 shots) with a 120 ns lifetime, and the on-axis laser emission (single shot) at a pump energy of $51 \mathrm{~mJ}$.

emission spectra as a function of pump power are shown in fig. 2 for the $B \rightarrow X$ transition. The vibrational progression observed in fluorescence $(2 a)$ disappears as the pump power is increased and at intermediatc powers only the strongest member of the progression is amplified ( $2 b$ ). At high pump powers (figs. $2 \mathrm{c}$ and $2 \mathrm{~d}$ ), a second amplified band on the high-energy side of the main laser transition is occasionally observed (2d). This secondary band is not part of the vibrational progression observed in the fluorescence spectrum which is dominated by $v^{\prime}=0 \rightarrow v^{\prime \prime}$ transitions. The spacing of $60 \mathrm{~cm}^{-1}$ between the two lasing lines indicates that it originates from the $v^{\prime}=1$ level of the B state. Evidently the gain is large enough such that relaxation via stimulated emission competes with nonradiative relaxation of the $v^{\prime}=1$ state. Estimates of the gain coefficients can be obtained from the observed line narrowing. The spectral distribution of the amplified intensity can be expressed as

$$
\begin{aligned}
& I(\lambda) \mathrm{d} \lambda=A \exp \left(-\Delta \lambda^{2} / 2 \sigma^{2}\right) \\
& \quad \times\left\{\exp \left[\alpha_{0} l \exp \left(-\Delta \lambda^{2} / 2 \sigma^{2}\right)\right]-1\right\} \mathrm{d} \lambda,
\end{aligned}
$$

in which $\alpha_{0}$ is the gain coefficient at line center, the band profile is assumed to be Gaussian of width $\sigma$, 
and $l$ represents the length of the active medium. The fwhm of the central band in the $B \rightarrow X$ progression decreases from its fitted value of 2.4 to $0.4 \mathrm{~nm}$ under strong amplification conditions. In this limit, the gain length product is well approximated by

$\alpha_{0} l=\frac{\ln 2}{1-\exp \left[-\ln 2\left(\Gamma / \Gamma^{\prime}\right)^{2}\right]}$,

in which $\Gamma$ and $\Gamma^{\prime}$ are fwhm for the amplified and fluorescence profiles respectively. The $\alpha_{0} l$ values at differcnt pump powers are collected in table 1 for both purple and green beams.

The fluorescence spectrum shown in fig. $3 a$ is modified when monitored along the cavity mirrors. In trace $b$ of fig. 3 the on-axis spectrum observed from the pump side is shown. The dielectric coating on this side is maximized for $99.9 \%$ reflection at 500 $\mathrm{nm}$, hence the blue degradation of $\mathrm{C} \rightarrow \mathrm{A}$ band. The observed structure on the red side is strictly due to the wavelength dependence of the mirror reflectivity. Increasing the pump power leads to lasing on the $B \rightarrow X$ transition (table 1). Upon increasing the pump energy to $50 \mathrm{~mJ}$, lasing on the $\mathrm{C} \rightarrow \mathrm{A}$ transition is observed as evidenced by the strong line narrowing (3c). The line is centered at the maximum of the gain profile. In accordance with the smaller $\gamma \sigma_{\mathrm{s}}$ value, the threshold for the $\mathrm{C} \rightarrow \mathrm{A}$ laser occurs at higher pump energies than that of the $B \rightarrow X$. At higher pump energies, the $B \rightarrow X$ laser saturates and the $C \rightarrow A$ laser intensity exceeds it due to the larger population in the $C$ state (fig. 3d). The gain-length product, $\alpha_{0} l$, calculated from the line narrowing (eq. (2)) reaches very large values of 30-50 (table 1).

It is informative to compare the observed laser parameters to the maximal values possible for this system under ideal conditions. For a dilution of $1: 3000$, if we assume that every Xe atom forms an excited $\mathrm{XeF}$ exciplex, the attainable number density, $N$, is $1 \times 10^{-19} \mathrm{~cm}^{-3}$. Assuming an optimal absorption probability of unity, a minimal pump flux of $50 \mathrm{~mJ}$ over the pump area of $1 \mathrm{~mm}^{2}$ is necessary for saturation of the centers. With the assumption of unit quantum efficiency, the expected maximal gain coefficients, $\alpha_{\max }=\gamma \sigma_{\mathrm{s}} N$, are 100 and $300 \mathrm{~cm}^{-1}$ for the $\mathrm{C} \rightarrow \mathrm{A}$ and $\mathrm{B} \rightarrow \mathrm{X}$ transitions, respectively (table 1 ). Given the resonator configuration, and losses due to scattering at the crystal interfaces, we may assume that the number of round trips in the cavity is small, and that the effective gain length is $\approx 1 \mathrm{~cm}$. This assumption is experimentally justified by the fact that the laser intensity is not sensitive to minor detuning of the cavity mirrors. It then follows that the observed values are very near the maximal possible for the present geometry (table 1 ).

This comparison has several important implications. Firstly, it requires that essentially all Xe atoms form XeF exciplexes. Thus the $\mathrm{F}_{2}$ dissociation has to be complete. In addition each $F$ atom has to reach a place adjacent to a Xe atom to form the exciplex. The last condition is by no means trivial since the mean separation between $X e$ and $F$ will be of the order of 15 nearest neighbor separations or $56 \AA$ for a dilution of $3 \times 10^{-4}$. Secondly, the overall quantum efficiency has to be close to unity: the deposited energy can be extracted as laser radiation with about unity efficiency. Thirdly, amplification of spontaneously emitted photons within the excited volume $N L / \tau \mathrm{c}$ in the amplification cone $\mathrm{d} \Omega$ should lead to a depletion of the excited state population at $\alpha_{0} l$ values of 10 for the $411 \mathrm{~nm}$ band and about 13 for the $536 \mathrm{~nm}$ band according to eq. (1). This predicted saturation is observed for the $411 \mathrm{~nm}$ band around pump powers of 15 to $25 \mathrm{~mJ}$ in agreement with the estimate (table 1). The strong depletion of the excited state by stimulated emission is further substantiated by measurements in the direction perpendicular to amplification. The side light intensity of the $530 \mathrm{~nm}$ band is reduced by at least a factor of 5 for an increase of the pump power from 18 to $43 \mathrm{~mJ}$. In addition the spectra show that a signiticant amount of the side light at high pump power is scattered amplified radiation and the real fluorescence content is even smaller. The system can indeed be described as an ideal four-level laser.

\section{Conclusions}

In summary, it has been shown that $\mathrm{Rg}^{+} \mathrm{X}^{-}$exciplex formation takes place in doubly doped rare gas crystals with high efficiency even at large dilution. Crystals of sufficient optical quality for lasing can be grown in a convenient way. Gain saturation is obtained at moderate optical pump powers. The deposited energy is extracted as laser radiation with a quantum efficiency near unity at stored energy 
densities of $10^{5} \mathrm{~J} / l$. An extension to shorter wavelengths seems to be feasible by choosing exciplexes with rare gases of lower and halogens with higher atomic weight. Such systems can compliment frequency mixing by which VUV radiation from 110 to $94 \mathrm{~nm}$ has been generated in $\mathrm{Kr}$ and Ar crystals [13]. Exciplex systems promise higher output powers.

\section{Acknowledgement}

This research was supported in part by the US Air Force Astronautics Laboratory under contract F0461 1-87-K-0024, and SPI under subcontract 1047 600-45. NS acknowledges the support of the Deutsche Forschungsgemeinschaft via SFB337 and the support of the University of California Institute for Surface and Interface Science during his stay at Irvine.

\section{References}

[1] H. Nahme, T. Kessler, R. Markus, M. Chergui and N. Schwentner, J. Luminescence 40/41 (1988) 821;

H. Nahme, Thesis, Freie I_Iniversität Rerlin (1988).
[2] H. Dubost, R. Charneau, M. Chergui and N. Schwentner, to be published.

[3] C.K. Rhodes, ed., Topics in applied physics, Vol. 30. Excimer lasers (Springer, Berlin, 1984).

[4] M.E. Fajardo, R. Withnall, J. Feld, F. Okada, W. Lawrence, L. Wiedeman and V.A. Apkarian, Laser Chem. 9 (1988) 1,

[5] M.E. Fajardo and V.A. Apkarian, J. Chem. Phys. 85 (1986) $5660 ; 89$ (1988) 4102.

[6] M.E. Fajardo and V.A. Apkarian, Chem. Phys. Letters 134 (1987) 51 .

[7] M. Shahid, H. Jara, H. Pummer, H. Egger and C.K. Rhodes, Opt. Letters 10 ( 1985 ) 448.

[8] T.R. Loree, R.R. Showalter, T.M. Johnson, B.S. Birmingham and W.M. Hughes, Opt. Letters 11 (1986) 570.

[9] L. Wiedeman, M.E. Fajardo and V.A. Apkarian, J. Phys. Chem. 92 (1988) 342.

[10] N. Schwentner and V.A. Apkarian, to be published.

[11] J. Goodman and L.E. Brus, J. Chem. Phys. 65 (1976) 3808.

[12] H. Okabe, Photochemistry of small molecules (Wiley, New York, 1978).

[13] G. Schilling, W.E. Ernst and N. Schwentner, Phys. Rev. Letters, submitted for publication. 